\title{
Brain Structural Covariance Network Topology in Remitted Posttraumatic Stress Disorder
}

\author{
Delin Sun ${ }^{1,2}$, Sarah L. Davis, ${ }^{1,2}$, Courtney C. Haswell ${ }^{1,2}$, Chelsea A. Swanson ${ }^{1,2}$, \\ Mid-Atlantic MIRECC Workgroup ${ }^{1+}$, Kevin S. LaBar ${ }^{1,2,3}$, John A. Fairbank ${ }^{1,3}$ \\ and Rajendra A. Morey ${ }^{1,2,3 *}$
}

'Department of Veteran Affairs (VA) Mid-Atlantic Mental Illness Research, Education and Clinical Center, Durham, NC, United States, ${ }^{2}$ Duke-UNC Brain Imaging and Analysis Center, Duke University, Durham, NC, United States,

${ }^{3}$ Department of Psychiatry and Behavioral Sciences, Duke University, Durham, NC, United States

\section{OPEN ACCESS}

Edited by:

Chantal Martin Soelch, University of Fribourg, Switzerland

Reviewed by: Mirko Manchia, Dalhousie University, Canada Lisa-Katrin Kaufmann, Universität Zürich, Switzerland

${ }^{*}$ Correspondence:

Rajendra A. Morey rajendra.morey@duke.edu

tThe contributors of the Mid-Atlantic MIRECC Workgroup are listed at the end of the article.

Specialty section:

This article was submitted to Mood and Anxiety Disorders, a section of the journal Frontiers in Psychiatry

Received: 22 September 2017 Accepted: 05 March 2018 Published: 29 March 2018

Citation:

Sun D, Davis SL, Haswell CC, Swanson CA, Mid-Atlantic MIRECC Workgroup, LaBar KS, Fairbank JA and Morey RA (2018) Brain

Structural Covariance

Network Topology in Remitted Posttraumatic Stress Disorder.

Front. Psychiatry 9:90. doi: 10.3389/fpsyt.2018.00090
Posttraumatic stress disorder (PTSD) is a prevalent, chronic disorder with high psychiatric morbidity; however, a substantial portion of affected individuals experience remission after onset. Alterations in brain network topology derived from cortical thickness correlations are associated with PTSD, but the effects of remitted symptoms on network topology remain essentially unexplored. In this cross-sectional study, US military veterans $(N=317)$ were partitioned into three diagnostic groups, current PTSD (CURR-PTSD, $N=101$ ), remitted PTSD with lifetime but no current PTSD (REMIT-PTSD, $N=35$ ), and trauma-exposed controls (CONTROL, $n=181$ ). Cortical thickness was assessed for 148 cortical regions (nodes) and suprathreshold interregional partial correlations across subjects constituted connections (edges) in each group. Four centrality measures were compared with characterize between-group differences. The REMIT-PTSD and CONTROL groups showed greater centrality in left frontal pole than the CURR-PTSD group. The REMIT-PTSD group showed greater centrality in right subcallosal gyrus than the other two groups. Both REMIT-PTSD and CURR-PTSD groups showed greater centrality in right superior frontal sulcus than CONTROL group. The centrality in right subcallosal gyrus, left frontal pole, and right superior frontal sulcus may play a role in remission, current symptoms, and PTSD history, respectively. The network centrality changes in critical brain regions and structural networks are associated with remitted PTSD, which typically coincides with enhanced functional behaviors, better emotion regulation, and improved cognitive processing. These brain regions and associated networks may be candidates for developing novel therapies for PTSD. Longitudinal work is needed to characterize vulnerability to chronic PTSD, and resilience to unremitting PTSD.

Keywords: posttraumatic stress disorder, remission, structural covariance network, cortical thickness, centrality

\section{INTRODUCTION}

Posttraumatic stress disorder (PTSD) is common, typically chronic, and associated with high rates of psychiatric comorbidity. In about $30-50 \%$ of patients with PTSD, marked symptoms persist after treatment (1) and severely impact quality of life $(2,3)$. However, a sizable minority of veterans who initially experience prominent symptoms of PTSD eventually experience remission $(4,5)$. 
A reassessment after 40 years of veterans who participated in the National Vietnam Veterans Readjustment revealed that while PTSD symptoms worsened for theater veterans with PTSD as a whole, there were $7.6 \%$ who showed significant clinical improvement (4). A similar large-scale longitudinal re-examination of the Vietnam era twins after 20 years identified $17 \%$ of theater veterans developed PTSD early and experienced early symptom remission, and 7.4\% who developed PTSD early and experienced late symptom remission (5). These results clearly highlight relapse and remission in PTSD $(6,7)$. Some recent studies have investigated the neural changes associated with the remission of PTSD in response to specific therapeutic interventions (8-10). However, our understanding of the associated neurostructural and network correlates of remission is still limited.

A wealth of research demonstrates that PTSD is associated with an array of functional and anatomical changes at specific anatomical loci in the brain, including amygdala, hippocampus, insula, ventral/dorsal medial/lateral prefrontal cortex, and anterior/posterior cingulate cortex (11-14). However, the human brain is organized into complex networks (15) supported by long range connections (16) that may be modified by exposure to traumatic events, precipitate alterations in network topology, and ultimately in behavior/symptoms $(17,18)$. Changes in network topology can be conveniently quantified by graph theoretical measures to understand changes in associated behavior and neuropsychiatric symptoms (i.e., dysfunctional behaviors) (19-21). Network architecture can be inferred from various neuroimaging methods including functional resting or task-based functional magnetic resonance imaging (fMRI), fiber connectivity from diffusion tensor imaging of white-matter tracts, and structural brain network derived from between-subject regional correlation of cortical thickness (22). Among them, cortical morphometric network analyses (23) are based on inferences about structural covariance between pairs of cortical regions that covary with respect to cortical thickness or subcortical regions that covary with respect to volume (24). The dependence between brain areas is postulated to derive from structural or functional associations between these regions $(22,24-26)$. The brain structural network method is relatively immune to a range of noisy components that accompany task-based and resting state fMRI, and purportedly reflects a highly choreographed developmental process of neuronal growth and migration throughout the cortical mantle $(24,27)$.

Mueller et al. (28) showed PTSD-associated alterations of structural brain networks, demonstrating an enhanced role of the left insula and right orbitofrontal cortices (betweenness centrality) and a diminished role of left orbitofrontal and anterior cingulate cortices (degree centrality) within the network. Centrality is a mathematical measure from graph theory that characterizes the importance of a particular region within a network of connections (edges) between brain regions (nodes). We investigated the network characteristics of remitted PTSD (lifetime but not current diagnosis) by comparing the centralities of structural networks to those in patients with current PTSD and traumaexposed control subjects without lifetime PTSD. Our goal was to identify structural network characteristics associated with PTSD and its remission. Such knowledge may help identify potential targets for interventions to ameliorate chronic PTSD by facilitating remission. The disorder is characterized by symptoms and behaviors that produce cognitive impairment particularly with attention and memory (29); difficulty with regulating emotions, particularly in response to threat and fear (30); avoidance, often in the form of social anxiety (31) and hypervigilance, among others (11). Thus, we hypothesized between-group differences of network centrality in cortical regions associated with cognitive function, emotion regulation, fear processing, social cognition, and inhibitory control, by focusing on the prefrontal cortex including anterior cingulate cortex, specifically subcallosal gyrus $(32,33)$, frontal pole, and superior frontal areas $(34,35)$ that have been associated with PTSD.

\section{MATERIALS AND METHODS}

\section{Participants}

Participants $(n=317)$ recruited from a repository (Mid-Atlantic MIRECC Post-Deployment Mental Health Repository, Durham, NC, USA) $(36,37)$ of Iraq and Afghanistan era military service members underwent structural MRI scans. Participants were screened for inclusion/exclusion criteria based on information available in the repository. Important exclusions included major axis I diagnosis (other than major depressive disorder or PTSD), contraindication to MRI, traumatic brain injury, substance dependence, neurological disorders, and age over 65 years. This study was carried out in accordance with the recommendations of the Institutional Review Boards at Duke University and the Durham VA Medical Center with written informed consent from all subjects. All subjects gave written informed consent in accordance with the Declaration of Helsinki. The protocol was approved by the Institutional Review Boards at Duke University and the Durham VA Medical Center. Participants previously completed questionnaires upon entering the repository that were available for this study to assess traumatic life events [Traumatic Life Events Questionnaire (TLEQ) (38)], combat exposure [Combat Exposure Scale (CES) (39)], depressive symptoms [Beck Depression Inventory-II (BDI-II) (40)], and serotonergic antidepressant medication use (Med_5HT). Diagnosis of PTSD was performed with the Clinician-Administered PTSD Scale to determine three groups (1) current PTSD who met diagnostic criteria based on symptoms experienced in the past month (CURR-PTSD; $n=101$ ), (2) lifetime PTSD but no current PTSD for participants who met diagnostic criteria before the last month but not since (REMIT-PTSD; $n=35$ ), and (3) those without PTSD who never met diagnostic criteria (CONTROL; $n=181$ ). Comorbid psychiatric diagnoses were ascertained upon entering the repository with the Structured Clinical Interview for DSM-IV (SCID). Childhood trauma (child-trauma) was coded from the number of trauma categories experienced before age 18 (e.g., physical abuse, sexual abuse, and serious accident) as reported in the TLEQ (41).

\section{MRI Acquisition and Analyses}

All images were acquired on 3-T scanners equipped with an 8 -channel headcoil. The majority $(90.6 \%)$ of images was acquired 
on two GE scanners using high-resolution T1-weighted wholebrain axial images with $1-\mathrm{mm}$ isotropic voxels with array spatial sensitivity encoding technique and fast spoiled gradient recall (3D-FSPGR). Image parameters were optimized for contrast between white matter, gray matter, and CSF on the (i) GE Discovery MR750 ( $n=156$, including 79 CONTROL, 21 REMIT-PTSD, and 56 CURR-PTSD) $\left(\right.$ TR/TE/flip angle $=7.484 \mathrm{~ms} / 2.984 \mathrm{~ms} / 12^{\circ}$, $\mathrm{FOV}=256 \mathrm{~mm}, 1 \mathrm{~mm}$ slice thickness, 166 slices, $256 \times 256$ matrix, 1 excitation) and (ii) GE Signa EXCITE ( $n=132$, including 80 CONTROL, 11 REMIT-PTSD, and 41 CURR-PTSD) $\left(\mathrm{TR} / \mathrm{TE} /\right.$ flip angle $=8.208 \mathrm{~ms} / 3.22 \mathrm{~ms} / 12^{\circ}, \mathrm{FOV}=256 \mathrm{~mm}$, 1 -mm slice thickness, 166 slices, $256 \times 256$ matrix, 1 excitation). The remaining images $(9.4 \%)$ were collected on a Philips Ingenia scanner $(n=29$, including 22 CONTROL, 3 REMITPTSD, and 4 CURR-PTSD) using higher in-plane resolution $0.9375 \mathrm{~mm} \times 0.9375 \mathrm{~mm} \times 1.0 \mathrm{~mm} 3 \mathrm{D}$ turbo field echo pulse sequence with contrast enhancement and SENSE (TR/TE/flip angle $=8.148 \mathrm{~ms} / 3.728 \mathrm{~ms} / 8^{\circ}, \mathrm{FOV}=240 \mathrm{~mm}, 1-\mathrm{mm}$ slice thickness, 170 slices, $256 \times 256$ matrix, 1 excitation). Chi-square test showed that the group distribution is independent of scanner $\left(\chi^{2}=8.725, \mathrm{df}=4, p=0.068\right)$. All T1 images were visually inspected to assure sufficient quality for automated segmentation and labeling, which were performed using the FreeSurfer image analysis suite (version 5.3.0; http://surfer.nmr.mgh.harvard.edu/) and its library tool recon-all. Details of FreeSurfer parcellations have been previously described (12, 42-45). Thickness measures were calculated for 148 cortical regions (74 per hemisphere) using the aparc.a2009s template (44) through the FreeSurfer software.

\section{Network Analyses}

We generated interregional partial correlation matrices for each participant group by calculating partial correlation coefficients for all regional pairings of cortical thickness across group members. The partial correlation between two regions represents their relationship after partialing out the effects of potential influences of age, sex, IQ, BDI-II, TLEQ, CES, child-trauma, and Med_5HT. These confounding factors have previously been associated with brain structural volumetry $(46,47)$. A threshold was imposed on the partial correlation matrices to create a binary graph with connections (edges) between regions $(19,48)$. Using the same threshold for group comparisons may give the results that reflect not only topological differences but also connectivity strength differences. We were interested in topological differences only and thus adopted group-specific thresholds to ensure that the graphs of all groups had an equal number of edges or wiring cost defined as the number of edges present divided by maximum possible number of edges. This method has been successfully utilized in the recent published works on maltreatment (48) and PTSD (28). We calculated the minimum wiring cost required to produce a fully connected network for each group and chose the largest minimum wiring cost (i.e., 0.4987, which was from the CONT group; minimum wiring costs were 0.0868 for REMITPTSD and 0.2075 for CURR-PTSD) across groups to derive the corresponding threshold for each group. This method ensured that all nodes were in the network while minimizing the number of redundant paths. We only kept the positive suprathreshold partial correlations in the networks due to the observation that only positive thickness correlations were mediated by direct fiber pathways (24). The network analyses were conducted using in-house Matlab (R2016b) scripts running on an iMac computer (macOS Sierra, version 10.12.6) (49).

\section{Centrality Measures}

A large array of network topology measures can be calculated for a given network and some provide very similar information to other measures. For ease of comparison to previous studies, we analyzed four types of centrality using Brain Connectivity Toolbox [BCT (50)]: (1) degree centrality-number of connections that a node has, (2) betweenness centrality-frequency with which a node falls between pairs of other nodes when traveling along their shortest interconnecting path, (3) closeness centrality-normalized number of steps required to access every other node from a given node in a network (adapted from the distance function in BCT), and (4) eigenvector centralitya spectral centrality measure based on the idea that the importance of a node is recursively related to the importance of the nodes associated with it. Graph theory postulates that nodes with high centrality play an important role in communication and information transfer within a network $(20,28$, 48). Naturally, the various centrality constructs are sometimes correlated but still reflect different aspects in nodal roles of a brain networks.

\section{Statistics}

The variance in the groups' measures was determined to be equal despite the disparate sample sizes of the three groups (51). We detected four cortical regions showing unequal variances (left lateral sulcus, right frontal pole, right anterior transverse temporal gyrus, and right temporal pole) through two-sample $F$ tests for equal variances (at the $1 \%$ significance level) and excluded them from further analysis. Furthermore, we tested the reliability of the centrality measures with the Jackknife resampling method (52) to calculate the $99 \%$ confidence interval (CI). The Jackknife resampling method has been successfully utilized in analyzing the network derived from partial correlations (48). Finally, to assess between-group differences, we employed permutation testing to compute the probability that the difference in centrality measures between two groups occurred by chance. The permutation testing was based on 10,000 network comparisons derived by randomly permuting the group label of subjects $(20,53)$. To control for type 1 error, we followed the procedure by Teicher et al. (48) and employed a more conservative threshold that deems a node differs in centrality between groups only if the permutation-derived $p$-values are $\leq 0.05$ for at least three of four centrality measures, which very conservatively reduces the odds of chance occurrence to $\leq 0.000125(0.05 \times 0.05 \times 0.05)$. The more conservative threshold was used because we were comparing among three groups while Teicher et al. (48) only compared between two groups. For a priori cortical regions, the nodal between-group differences were considered significant when the permutation-derived $p$-values are $\leq 0.05$ for at least two of four centrality measures. The statistical analyses were conducted using in-house Matlab scripts. The code for permutation testing was modified from the GRETNA toolbox (54). 


\section{RESULTS}

\section{Demographic and Clinical Characteristics}

Participants' age, sex, and other demographic and clinical information are summarized in Table 1. The three groups did not significantly differ with respect to age and gender.

\section{Centrality Measures}

The centrality measures of all nodes were within their corresponding $99 \%$ CI, supporting the reliability of the analyses. Significant between-group differences of nodal centrality were identified in several cortical regions. Specifically, centrality between-group differences were detected in anterior cingulate cortex, frontal pole and superior frontal areas (see Figure 1).

TABLE 1 | Demographic information.

\begin{tabular}{|c|c|c|c|c|c|c|}
\hline Test & \multicolumn{3}{|c|}{ Mean (SD) ${ }^{\mathrm{a}}$} & \multicolumn{3}{|c|}{$t$-Statistic $(p \text {-value })^{\mathrm{b}}$} \\
\hline Sex & $28(7)$ & $87(14)$ & $145(36)$ & 0.000 (0.988) & $0.750(0.386)$ & $-1.615(0.204)$ \\
\hline IQ & $99.6(11.6)$ & 95.8 (12.3) & $101.8(9.5)$ & $-1.174(0.242)$ & 1.500 (0.136) & $-4.440(0.001)$ \\
\hline BDI-II & $10.4(7.8)$ & $22.2(12.1)$ & $5.1(7.8)$ & $3.648(<0.001)$ & $-5.336(<0.001)$ & $14.386(<0.001)$ \\
\hline Child-trauma & $1.0(1.0)$ & $0.7(1.0)$ & $0.4(0.8)$ & $3.168(0.002)$ & $1.161(0.248)$ & 2.371 (0.019) \\
\hline Med_5HT & $5(29)$ & $51(49)$ & 4 (172) & $-10.738(0.001)$ & $13.740(<0.001)$ & $-94.888(<0.001)$ \\
\hline AUDIT & $4.6(3.5)$ & $4.2(5.4)$ & $2.8(3.4)$ & $2.618(0.010)$ & $0.362(0.718)$ & $2.414(0.017)$ \\
\hline CAPS_curr & $19.4(13.9)$ & $68.2(22.4)$ & $7.0(11.1)$ & $5.451(<0.001)$ & $-11.437(<0.001)$ & $29.119(<0.001)$ \\
\hline CAPS_life & $63.5(21.0)$ & 83.5 (29.6) & $14.7(15.8)$ & $14.885(<0.001)$ & $-3.462(0.001)$ & $24.160(<0.001)$ \\
\hline DAST & $0.7(1.5)$ & $1.1(2.5)$ & $0.4(0.8)$ & $1.404(0.162)$ & $-0.923(0.357)$ & $3.295(0.001)$ \\
\hline
\end{tabular}

"Values outside/inside brackets are number of either males/females for "sex" or yes/no for "Med_5HT."

"Statistical values are from chi-square tests for "sex" and "med_5HT."

CONTROL, control group with trauma exposure; CURR-PTSD, current PTSD group; REMIT-PTSD, lifetime but no current PTSD group; IQ, intelligence quotient; BDI-II; Beck Depression Inventory-II; TLEQ, trauma life events questionnaire; CES, Combat Exposure Scale; Child-trauma, categories of trauma exposure as child/adolescent; Med_5HT, serotonergic medication; AUDIT, Alcohol Use Disorders Identification Test; CAPS_curr, Clinician-Administered PTSD Scale reflecting symptoms in the last 30 days; CAPS_life, Clinician-Administered PTSD Scale reflecting symptoms in the worst 30-day period of subject's life; DAST, Drug Abuse Screening Test; DTS, Davidson Trauma Scale; PTSD; posttraumatic stress disorder; TLEQ; Traumatic Life Events Questionnaire.

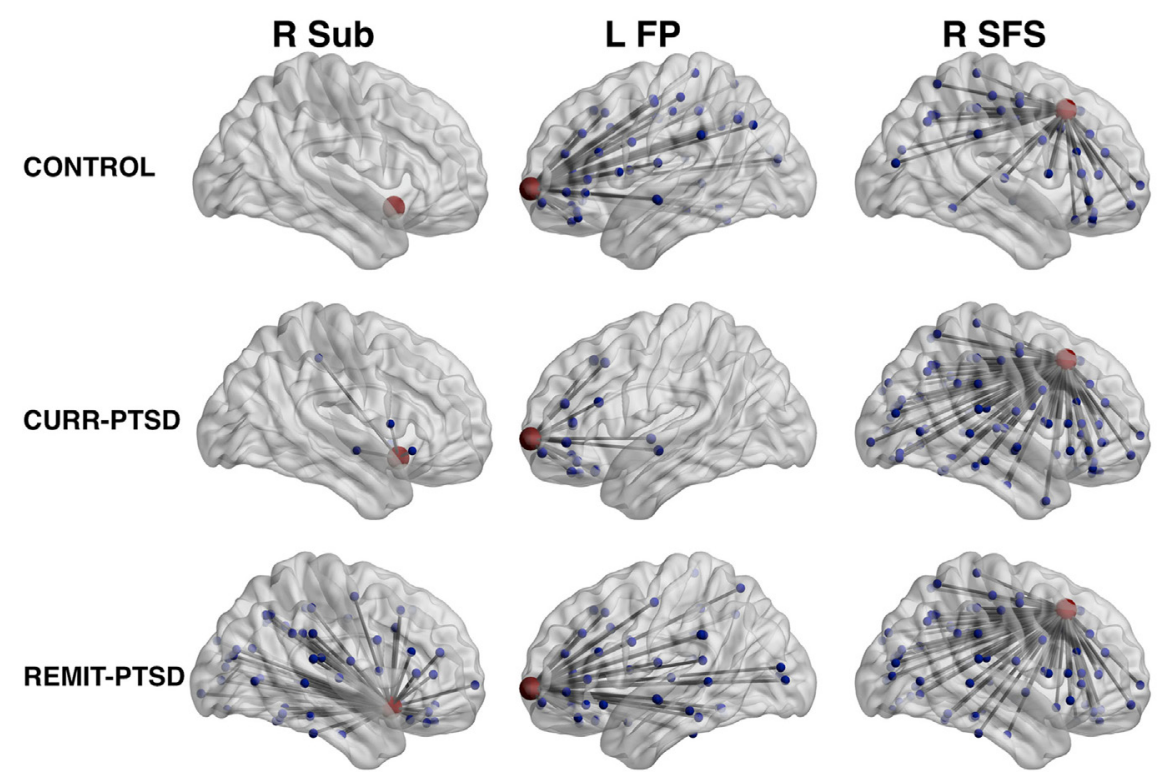

FIGURE 1 | Between-group differences of centrality measures. Left column, REMIT-PTSD (lifetime but no current PTSD group) group showed larger centrality in right subcallosal gyrus (R Sub) than both CURR-PTSD (current PTSD group) and CONTROL (control group with trauma exposure) groups. Middle column, both REMIT-PTSD and CONTROL groups showed larger centrality in left frontal pole (L FP) than CURR-PTSD group. Right column, both REMIT-PTSD and CURR-PTSD groups showed larger centrality in right superior frontal sulcus (R SFS) than CONTROL group. For observation purpose, the regions of interest (red), their connected nodes (blue), and the connections (gray) were shown. Node size was scaled by degree centrality. 


\section{REMIT-PTSD Versus CONTROL}

As shown in Table 2, REMIT-PTSD patients (versus CONTROL) showed smaller centrality in left paracentral lobule and sulcus and bilateral precentral gyrus. They also showed larger centrality in right subcallosal gyrus, vertical ramus of the anterior segment of the lateral sulcus, superior segment of the circular sulcus of the insula, superior frontal sulcus, and anterior occipital sulcus.

\section{REMIT-PTSD Versus CURR-PTSD}

As shown in Table 3, REMIT-PTSD (versus CURR-PTSD) patients showed smaller centrality in left precentral gyrus, right precuneus, right middle temporal gyrus, and right inferior frontal sulcus. They also showed larger centrality in left transverse frontopolar gyri and sulci, left posterior-dorsal part of the cingulate gyrus, right lateral occipito-temporal gyrus, and right subcallosal area.

\section{CURR-PTSD Versus CONTROL}

As shown in Table 4, CURR-PTSD patients (versus CONTROL) showed smaller centrality in left transverse frontopolar gyri and sulci, left posterior-dorsal part of the cingulate gyrus, left postcentral gyrus, left inferior segment of the circular sulcus of the insula, and right anterior transverse collateral sulcus. They also showed larger centrality in left sulcus intermedius primus (of Jensen), right precuneus, right inferior/middle/superior frontal sulcus, right lateral orbital sulcus, and right inferior part of the precentral sulcus.

\section{DISCUSSION}

We characterized neuroanatomical networks defined by cortical thickness correlations for comparison between groups with current PTSD, remitted PTSD, and trauma-exposed controls. Consistent with our a priori hypotheses, significant differences between REMIT-PTSD and CURR-PTSD as well as CONTROL participants were detected in the right subcallosal gyrus, frontal pole (left transverse frontopolar gyri and sulci) and superior frontal sulcus. Network connectivity of the frontal pole in REMIT-PTSD appeared to assume a connectivity profile that is consistent with trauma-exposed controls, whereas the CURR-PTSD group had altered frontopolar network connectivity. On the other hand, the REMIT-PTSD group demonstrated brain network architecture at the superior frontal sulcus that was consistent with CURR-PTSD but clearly altered compared with CONTROL despite the lack of PTSD symptoms. A precise neurobiological interpretation of the between-group differences in the covariance of cortical thickness between regions is yet unclear. It is possible that the correlation strength increases between regions that are concurrently affected by disorder (or recovery) processes, which are perhaps due to loss of (or new) input from directly affected regions, and the

TABLE 2 | Centrality between-group comparisons: REMIT-PTSD (lifetime but no current PTSD group) versus CONTROL (control group with trauma exposure).

\begin{tabular}{|c|c|c|c|c|c|}
\hline No. & Area & Degree & Betweenness & Closeness & Eigenvector \\
\hline \multicolumn{6}{|c|}{ REMIT-PTSD < CONTROL } \\
\hline 3 & L Paracentral lobule and sulcus & $41 / 91$ & 14.0/63.0 & $0.639 / 0.803$ & $0.046 / 0.099$ \\
\hline 29 & L Precentral gyrus & $64 / 117$ & $31.5 / 194.8$ & $0.718 / 0.891$ & $0.074 / 0.119$ \\
\hline 29 & R Precentral gyrus & $67 / 112$ & $60.8 / 134.7$ & $0.728 / 0.874$ & $0.073 / 0.115$ \\
\hline \multicolumn{6}{|c|}{ REMIT-PTSD > CONTROL } \\
\hline 32 & R Subcallosal gyrus* & $67 / 0$ & $53.9 / 0.0$ & $0.728 / 0.000$ & $0.075 / 0.000$ \\
\hline 40 & $\mathrm{R}$ Vertical ramus of the anterior segment of the lateral sulcus & $88 / 7$ & $111.7 / 0.0$ & $0.799 / 0.509$ & $0.095 / 0.008$ \\
\hline 49 & R Superior segment of the circular sulcus of the insula & $120 / 108$ & $264.4 / 246.4$ & $0.908 / 0.861$ & $0.124 / 0.105$ \\
\hline 54 & R Superior frontal sulcus* & $101 / 58$ & $126.6 / 19.8$ & $0.844 / 0.690$ & $0.111 / 0.063$ \\
\hline 59 & R Anterior occipital sulcus & $110 / 70$ & $210.7 / 42.6$ & $0.874 / 0.731$ & $0.116 / 0.076$ \\
\hline
\end{tabular}

At least three of four (two of four in a priori areas marked with *) centrality measures showed significant between-group differences in these areas.

"No." is the label of cortical area in the aparc.a2009s template (44).

$L$, left; $R$, right.

TABLE 3 | Centrality between-group comparisons: REMIT-PTSD (lifetime but no current PTSD group) versus CURR-PTSD (current PTSD group).

\begin{tabular}{|c|c|c|c|c|c|}
\hline No. & Area & Degree & Betweenness & Closeness & Eigenvector \\
\hline \multicolumn{6}{|c|}{ REMIT-PTSD < CURR-PTSD } \\
\hline 29 & L Precentral gyrus & $64 / 105$ & $31.5 / 108.8$ & $0.718 / 0.857$ & $0.074 / 0.112$ \\
\hline 30 & R Precuneus & $80 / 119$ & $59.5 / 202.1$ & $0.772 / 0.905$ & $0.090 / 0.121$ \\
\hline 38 & R Middle temporal gyrus & $71 / 112$ & $76.0 / 137.9$ & $0.741 / 0.881$ & $0.074 / 0.118$ \\
\hline 52 & R Inferior frontal sulcus & $66 / 114$ & $41.0 / 159.0$ & $0.724 / 0.888$ & $0.075 / 0.118$ \\
\hline \multicolumn{6}{|c|}{ REMIT-PTSD > CURR-PTSD } \\
\hline 5 & L Transverse frontopolar gyri and sulci & $65 / 18$ & $46.0 / 1.2$ & $0.721 / 0.543$ & $0.071 / 0.011$ \\
\hline 9 & L Posterior-dorsal part of the cingulate gyrus & $94 / 45$ & $154.5 / 18.5$ & $0.820 / 0.653$ & $0.097 / 0.044$ \\
\hline 21 & R Lateral occipito-temporal gyrus & $116 / 83$ & $205.2 / 97.3$ & $0.895 / 0.782$ & $0.124 / 0.081$ \\
\hline 32 & R Subcallosal area* & $67 / 8$ & $53.9 / 4.0$ & $0.728 / 0.518$ & $0.075 / 0.006$ \\
\hline
\end{tabular}

At least three of four (two of four in a priori areas marked with *) centrality measures showed significant between-group differences in these areas.

"No." is the label of cortical area in the aparc.a2009s template (44).

$L$, left; $R$, right. 
TABLE 4 | Centrality between-group comparisons: CURR-PTSD (current PTSD group) versus CONTROL (control group with trauma exposure).

\begin{tabular}{|c|c|c|c|c|c|}
\hline No. & Area & Degree & Betweenness & Closeness & Eigenvector \\
\hline \multicolumn{6}{|c|}{ CURR-PTSD $<$ CONTROL } \\
\hline 5 & L Transverse frontopolar gyri and sulci & $18 / 68$ & $1.2 / 56.6$ & $0.543 / 0.724$ & $0.011 / 0.073$ \\
\hline 9 & L Posterior-dorsal part of the cingulate gyrus & $45 / 104$ & $18.5 / 241.0$ & $0.653 / 0.847$ & $0.044 / 0.104$ \\
\hline 28 & L Postcentral gyrus & $57 / 92$ & $7.7 / 52.4$ & $0.686 / 0.806$ & $0.063 / 0.101$ \\
\hline 48 & $\mathrm{~L}$ Inferior segment of the circular sulcus of the insula & $51 / 105$ & $107.4 / 151.0$ & $0.673 / 0.850$ & $0.042 / 0.105$ \\
\hline 50 & R Anterior transverse collateral sulcus & $19 / 62$ & $1.0 / 26.1$ & $0.557 / 0.704$ & $0.023 / 0.068$ \\
\hline \multicolumn{6}{|c|}{ CURR-PTSD > CONTROL } \\
\hline 55 & L Sulcus intermedius primus (of Jensen) & $47 / 11$ & $5.9 / 0.0$ & $0.653 / 0.514$ & $0.052 / 0.012$ \\
\hline 30 & R Precuneus & $119 / 91$ & $202.1 / 48.0$ & $0.905 / 0.803$ & $0.121 / 0.100$ \\
\hline 52 & R Inferior frontal sulcus & $114 / 90$ & 159.0/101.0 & 0.888/0.799 & $0.118 / 0.095$ \\
\hline 53 & R Middle frontal sulcus & 99/31 & 150.4/13.2 & $0.837 / 0.598$ & $0.100 / 0.029$ \\
\hline 54 & R Superior frontal sulcus* & $115 / 58$ & $339.8 / 19.8$ & $0.891 / 0.690$ & $0.114 / 0.063$ \\
\hline 62 & R Lateral orbital sulcus & $62 / 14$ & $50.7 / 1.2$ & $0.711 / 0.536$ & $0.068 / 0.014$ \\
\hline 68 & $\mathrm{R}$ Inferior part of the precentral sulcus & $112 / 81$ & $183.5 / 75.3$ & $0.881 / 0.769$ & $0.117 / 0.089$ \\
\hline
\end{tabular}

At least three of four (two of four in a priori areas marked with *) centrality measures showed significant between-group differences in these areas.

"No." is the number of cortical area in the aparc.a2009s template (44).

$L$, left; $R$, right.

correlation strength decreases between affected and unaffected regions (28). Our result is consistent with previous findings that frontopolar cortical thinning is associated with increased PTSD symptom severity (35) but to the best of our knowledge this is the first published report to directly compare remitted PTSD to current PTSD, and trauma-exposed controls.

The REMIT-PTSD and CONTROL groups showed larger centrality in the left frontal pole than the CURR-PTSD group, thereby implicating this area in current PTSD symptoms. We posited that lower frontal pole centrality in CURR-PTSD is associated with non-synchronized cortical thickness changes with regions involving cognitive, social, emotional, and most notably selfreferential processing (55). This idea is consistent with previous findings that PTSD patients showed altered functional connectivity within brain structures associated with default model network (DMN), which plays a key role in self-referential processing and social cognition (56). The DMN consists of several areas including the anterior medial cortices. Interestingly, REMIT-PTSD and CONTROL groups did not differ in left frontopolar centrality. Clinically, PTSD associates with disturbances in self-referential processing whereby trauma undermines a sense of adaptive selfagency (57). Other closely related cognitive constructs of selfreference that are negatively impacted by PTSD include shame, guilt, self-blame, and a fragmented self-image (58-60). Key neural correlates of these self-referential cognitions are situated in the frontal pole $(12,55)$. Accordingly, PTSD exhibits functional differences in the frontopolar area for behavioral challenge tasks (11) and during the resting state (61). It is yet unclear whether the higher centrality in REMIT-PTSD (versus CURR-PTSD) patients reflects a recovery of network topology back to a configuration that was present before onset of PTSD, or a compensatory reorganization of network topology to comparable centrality at the frontal pole. These interpretations will require testing in future studies on the role of the frontal pole in remitted and current PTSD and could be explored as a potential target for therapeutic intervention. A recent study using transcranial direct current stimulation (tDCS) has already targeted ventromedial prefrontal cortex for the treatment of PTSD (62). Future clinical intervention on PTSD may utilize interventions including tDCS and deep brain stimulation targeting frontal pole.

The REMIT-PTSD group showed larger centrality in right subcallosal area than CURR-PTSD and CONTROL groups, suggesting the role of subcallosal area in representing the specific status of remitted PTSD patients. The subcallosal area is located in subgenual anterior cingulate cortex and is strongly associated with emotion processing (63). Reduced volume in this area was reported in combat-related PTSD patients compared with trauma-exposed controls (32) and cortical thinning in PTSD remitters compared with non-remitters following treatment with prolonged exposure and trauma-exposed healthy volunteers (9). The subcallosal area also connects with several cortical and subcortical structures including prefrontal cortex, cingulate cortex, amygdala, and hippocampus (64), which have been implicated in PTSD based on anatomical and functional changes $(11,12,14)$. However, our findings demonstrated that concurrent changes in cortical thickness in the subcallosal area and its structurally correlated regions were present only in the REMIT-PTSD group, but not in CURR-PTSD (compared with CONTROL). It is possible that a compensatory re-organization of network topology with larger centrality at right subcallosal area contributes to the resilience after experiencing PTSD symptoms by promoting symptom remission. However, we cannot exclude the possibility that some patients have special network topology with greater centrality in subcallosal area that helps with remission from PTSD symptoms. Indeed, US Special Forces who demonstrated resilience in the face of severe trauma have enhanced subcallosal activity during expectation of reward (65).

The REMIT-PTSD and CURR-PTSD groups showed larger centrality in right superior frontal sulcus than the CONTROL group, possibly implicating an enduring marker of prior PTSD even when symptoms remit. Our findings are consistent with previous reports that reduced cortical thickness in the superior frontal area is associated with PTSD symptom severity $(34,35)$. The superior frontal regions (covering both superior frontal sulcus and gyrus) have been associated with high-level cognitive 
functions such as working memory and inhibition control $(66,67)$, which contribute to downregulating the personal relevance of the negative events (68). Structural and functional connectivity methods show that the superior frontal area consists of subregions that connect with nodes of the cognitive executive network (including middle and inferior frontal gyrus), default mode network (including anterior and middle cingulate cortex), and motor control network (including precentral gyrus, caudate, thalamus, and frontal operculum) (69). Thus, our results suggest that a high centrality in the right superior frontal sulcus represents lifetime PTSD-related neural changes in brain areas typically associated with executive, motor, and self-processing. Given that PTSD is a chronic relapsing and remitting disorder $(4,70)$, it is possible that some neural changes persist following symptom remission and may serve as a biomarker of possible future relapse. It is possible, although unlikely, that these network connectivity changes are precipitated by trauma in certain individuals given the absence of this neural configuration in the trauma-exposed group. However, given that childhood trauma that is associated with lasting brain changes (71), we cannot exclude the possible influence of higher childhood trauma scores in the CURR-PTSD and REMIT-PTSD patients.

Our study provided comparable results to previous structural network analyses on individuals with PTSD. We found larger centrality in right lateral orbital sulcus in individuals with CURR-PTSD than in CONTROL subjects, which is consistent with Mueller et al. (28) who report larger betweenness centrality in the right orbitofrontal area of veterans with PTSD obtained from either a whole-brain network analysis or a network analysis restricted to prefrontal-limbic areas. These convergent findings suggest that larger centrality in right orbitofrontal area is associated with PTSD symptoms. Previous studies have detected reduced gray matter volume/concentration in orbitofrontal cortex in patients with either PTSD (72) or depression (73). In line with these results, the larger centrality in right orbitofrontal area in CURR-PTSD patients may reflect reduced gray matter in both right orbitofrontal area and its connected regions. No significant differences of centrality in this area were detected between REMIT-PTSD group and the other groups.

Various factors influence remission, including the trauma type, chronicity of trauma exposure, comorbid substance use, and others (74). In a large meta-analysis of 42 studies with a total of 81,642 participants, $44 \%$ of individuals with PTSD experienced spontaneous remission (without specific treatment) at a mean follow-up duration of 40 months (75). Given that we did not specifically assess the antecedents of PTSD remission, it is likely that the present sample was comprised of a mix of patients who experienced spontaneous remission, pharmacotherapeutic remission, psychotherapeutic remission, and some combination thereof. While spontaneous remission is perhaps of particular interest since it represents a form of resilience to PTSD-onset following trauma exposure, the ability to remit in response to treatment is equally interesting because it represents its own form of resilience when contrasted with patients who suffer chronic persistent PTSD and are refractory to treatment. Thus, our REMIT-PTSD sample represents a resilient sample, albeit heterogeneous, composed of a mix of individuals who recovered spontaneously and others who responded to treatment. Future research will be required to dissect the cortical network changes that are unique to each of the various subtypes of remitted patients.

The CURR-PTSD patients showed more severe depression and PTSD symptoms than REMIT-PTSD individuals, suggesting that the differences between CURR-PTSD and REMIT-PTSD patients might be confounded with the severity of depression and PTSD. However, a recent study showed that high depression severity is accompanied with greater centrality in ventral medial prefrontal cortex and posterior cingulate cortex, and smaller centrality in temporal areas and middle/ inferior frontal areas (76), which are largely contrary to our findings of the comparison between CURR-PTSD and REMITPTSD patients. Therefore, depression severity should not be a confounding factor when explaining our findings. On the other hand, veterans with PTSD were found to be associated with decreased degree centrality in medial orbital frontal areas and rostral cingulate cortex (28), consistent with our contrast between CURR-PTSD and REMIT-PTSD patients. We thus cannot reject the hypothesis that patients with less severe PTSD symptoms are more amenable to remission. Future studies on CURR-PTSD and REMIT-PTSD patients who are matched for lifetime PTSD scores should further address this issue.

\section{Limitations}

There are several limitations in this study. First, our analysis was based on the large-scale covariance of cortical thickness. Some subcortical regions important to PTSD, such as amygdala and hippocampus $(14,77)$, were not considered. Recent studies have included both cortical thickness and subcortical volumes in structural network analyses (28) although the reliability of this method still needs validation. Future studies should delineate the role of subcortical structures in the brain network of REMIT-PTSD patients. Second, the role of individual differences on network characteristics is unclear, given that a network is defined at the group level. A new approach for investigating cortical thickness networks is needed to investigate the relationship between cortical thickness-based network attributes and individual characteristics (e.g., age and gender). An single-subject gray matter graph method has been developed in recent years $(78,79)$ and should be utilized in future studies of PTSD and remission. Furthermore, other connectivity analyses methods such as resting state fMRI, based on interregional relationship across measures within each subject, may complement our understanding of the effect of individual difference in network topography. Third, our study utilized a cross-sectional design, which limits inferences about the causal relationships between PTSD remission and cortical thickness network. Future longitudinal studies should fill this gap and explore the relationship between brain network characteristics and treatment outcomes. Longitudinal studies $(4,5)$ assessing PTSD at two time points to assess the course of illness would be more reliable and robust than a cross-sectional approach because it does not rely on patients' memories of prior symptoms from the distant past. Last but not least, it is a challenge to estimate the sample size appropriate for a structural covariance network analysis. Conventional 
power analysis makes comparisons of the group means and variances of the same measures from individuals. By contrast, in our study, no centrality measures can be calculated at the individual subject level that can be pooled for group means and variances. Moreover, connection topology at each node determines the centrality, which means that each graph (group) has 148 relevant centrality measures to consider in any power or sample size calculation. The centrality at each node is not available as a mean and pooled variance that is derived from all subjects in that group. Furthermore, the large number of nodes at which centrality is compared between groups is a separate but related concern about adjusting power for multiple comparison testing. At this stage the field of connectomics, which is based on graph theoretical measures is yet grappling to develop appropriate corrections for multiple comparison testing that may be deployed on graph theoretical measures, and is even further from reaching consensus on best practices (80).

\section{Conclusion}

Our methods and results advance our understanding of the network configuration defined by structural relationships within REMIT-PTSD patients and may offer therapeutic targets for PTSD. Cortical thickness networks, specifically centrality of the right subcallosal gyrus, left frontal pole, and right superior frontal sulcus, differ between remitted PTSD, current PTSD diagnosis, and trauma exposure without PTSD. Our findings in REMITPTSD show enhanced structural connectivity that may represent a marker of resilience by promoting symptom remission through a recovery of network topology to a premorbid configuration or a compensatory re-organization of network topology with comparable centrality features.

\section{MID-ATLANTIC MIRECC WORKGROUP}

The Mid-Atlantic MIRECC Workgroup includes the following contributors: Jean C. Beckham, Mira Brancu, Patrick S. Calhoun, Eric Dedert, Eric B. Elbogen, Kimberly T. Green, Robin A. Hurley, Jason D. Kilts, Nathan Kimbrel, Angela Kirby, Christine E. Marx, Gregory

\section{REFERENCES}

1. Bradley R, Greene J, Russ E, Dutra L, Westen D. A multidimensional meta-analysis of psychotherapy for PTSD. Am J Psychiatry (2005) 162:214-27. doi:10.1176/appi.ajp.162.2.214

2. Kessler RC. Posttraumatic stress disorder: the burden to the individual and to society. J Clin Psychiatry (2000) 61:4-12; discussion 13-4.

3. Calhoun PS, Beckham JC, Bosworth HB. Caregiver burden and psychological distress in partners of veterans with chronic posttraumatic stress disorder. J Trauma Stress (2002) 15:205-12. doi:10.1023/A:1015251210928

4. Marmar CR, Schlenger W, Henn-Haase C, Qian M, Purchia E, Li M, et al. Course of posttraumatic stress disorder 40 years after the Vietnam war findings from the national Vietnam veterans longitudinal study. JAMA Psychiatry (2015) 72:875-81. doi:10.1001/jamapsychiatry.2015.0803

5. Magruder KM, Goldberg J, Forsberg CW, Friedman MJ, Litz BT, Vaccarino V, et al. Long-term trajectories of PTSD in Vietnam-era veterans: the course and consequences of PTSD in twins. J Trauma Stress (2016) 29:5-16. doi:10.1002/ jts. 22075
McCarthy, Scott D. McDonald, Marinell Miller-Mumford, Scott D. Moore, Rajendra A. Morey, Jennifer C. Naylor, Treven C. Pickett, Jared Rowland, Jennifer J. Runnals, Cindy Swinkels, Steven T. Szabo, Katherine H. Taber, Larry A. Tupler, Elizabeth E. Van Voorhees, H. Ryan Wagner, Richard D. Weiner, Ruth E. Yoash-Gantz.

\section{ETHICS STATEMENT}

All subjects gave written informed consent in accordance with the Declaration of Helsinki. The protocol was approved by the Institutional Review Boards at Duke University and the Durham VA Medical Center.

\section{AUTHOR CONTRIBUTIONS}

All of the authors made substantial contributions to the conception or design of the work; the acquisition, analysis, or interpretation of data for the work; drafting the work or revising it critically for important intellectual content; final approval of the version to be published; and agreement to be accountable for all aspects of the work in ensuring that questions related to the accuracy or integrity of any part of the work are appropriately investigated and resolved.

\section{FUNDING}

This research received financial support from the Veterans Health Affairs (VHA) Mid-Atlantic Mental Illness Research Education and Clinical Center (MIRECC) and the Office of Research and Development (5I01CX000748-01, 5I01CX000120-02). Additional financial support was provided by the National Institute for Neurological Disorders and Stroke (R01NS086885-01A1). Dr. Dedert was funded by a Department of Veterans Affairs Clinical Science Research and Development Career Development Award (IK2CX000718). The VHA played no role in the design and conduct of the study; collection, management, analysis, and interpretation of the data; preparation, review, or approval of the manuscript; and decision to submit the manuscript for publication.

6. Breslau N, Kessler RC, Chilcoat HD, Schultz LR, Davis GC, Andreski P. Trauma and posttraumatic stress disorder in the community - the 1996 Detroit area survey of trauma. Arch Gen Psychiatry (1998) 55:626-32. doi:10.1001/ archpsyc.55.7.626

7. Chapman C, Mills K, Slade T, Mcfarlane AC, Bryant RA, Creamer M, et al. Remission from post-traumatic stress disorder in the general population. Psychol Med (2012) 42:1695-703. doi:10.1017/S0033291711002856

8. Simmons AN, Norman SB, Spadoni AD, Strigo IA. Neurosubstrates of remission following prolonged exposure therapy in veterans with posttraumatic stress disorder. Psychother Psychosom (2013) 82:382-9. doi:10.1159/000348867

9. Helpman L, Papini S, Chhetry BT, Shvil E, Rubin M, Sullivan GM, et al. PTSD remission after prolonged exposure treatment is associated with anterior cingulate cortex thinning and volume reduction. Depress Anxiety (2016) 33:384-91. doi:10.1002/da.22471

10. van Rooij SJ, Kennis M, Vink M, Geuze E. Predicting treatment outcome in PTSD: a longitudinal functional MRI study on trauma-unrelated emotional processing. Neuropsychopharmacology (2016) 41:1156-65. doi:10.1038/npp. 2015.257 
11. Morey RA, Dolcos F, Petty CM, Cooper DA, Hayes JP, Labar KS, et al. The role of trauma-related distractors on neural systems for working memory and emotion processing in posttraumatic stress disorder. J Psychiatr Res (2009) 43:809-17. doi:10.1016/j.jpsychires.2008.10.014

12. Morey RA, Gold AL, Labar KS, Beall SK, Brown VM, Haswell CC, et al. Amygdala volume changes in posttraumatic stress disorder in a large casecontrolled veterans group. Arch Gen Psychiatry (2012) 69:1169-78. doi:10.1001/ archgenpsychiatry.2012.50

13. Brown VM, Labar KS, Haswell CC, Gold AL, Mccarthy G, Morey RA, et al. Altered resting-state functional connectivity of basolateral and centromedial amygdala complexes in posttraumatic stress disorder. Neuropsychopharmaco$\log y$ (2014) 39:351-9. doi:10.1038/npp.2013.197

14. Morey RA, Haswell CC, Hooper SR, De Bellis MD. Amygdala, hippocampus, and ventral medial prefrontal cortex volumes differ in maltreated youth with and without chronic posttraumatic stress disorder. Neuropsychopharmacology (2016) 41:791-801. doi:10.1038/npp.2015.205

15. Di Martino A, Fair DA, Kelly C, Satterthwaite TD, Castellanos FX, Thomason ME, et al. Unraveling the miswired connectome: a developmental perspective. Neuron (2014) 83:1335-53. doi:10.1016/j.neuron.2014.08.050

16. Zhang K, Sejnowski TJ. A universal scaling law between gray matter and white matter of cerebral cortex. Proc Natl Acad Sci U S A (2000) 97:5621-6. doi:10.1073/pnas.090504197

17. Morey RA, Lancaster SC, Haswell CC. Trauma re-experiencing symptoms modulate topology of intrinsic functional networks. Biol Psychiatry (2015) 78:156-8. doi:10.1016/j.biopsych.2015.06.001

18. Spielberg JM, Mcglinchey RE, Milberg WP, Salat DH. Brain network disturbance related to posttraumatic stress and traumatic brain injury in veterans. Biol Psychiatry (2015) 78:210-6. doi:10.1016/j.biopsych.2015.02.013

19. Bassett DS, Bullmore E, Verchinski BA, Mattay VS, Weinberger DR, MeyerLindenberg A. Hierarchical organization of human cortical networks in health and schizophrenia. JNeurosci (2008) 28:9239-48. doi:10.1523/ JNEUROSCI.1929-08.2008

20. He Y, Chen Z, Evans A. Structural insights into aberrant topological patterns of large-scale cortical networks in Alzheimer's disease. J Neurosci (2008) 28:4756-66. doi:10.1523/JNEUROSCI.0141-08.2008

21. Yao ZJ, Zhang YC, Lin L, Zhou YA, Xu CL, Jiang TZ, et al. Abnormal cortical networks in mild cognitive impairment and Alzheimer's disease. PLoS Comput Biol (2010) 6:e1001006. doi:10.1371/journal.pcbi.1001006

22. He Y, Evans A. Graph theoretical modeling of brain connectivity. Curr Opin Neurol (2010) 23:341-50. doi:10.1097/WCO.0b013e32833aa567

23. Sporns O. Networks of the Brain. Cambridge, MA: MIT Press (2011).

24. Gong GL, He Y, Chen ZJ, Evans AC. Convergence and divergence of thickness correlations with diffusion connections across the human cerebral cortex. Neuroimage (2012) 59:1239-48. doi:10.1016/j.neuroimage.2011.08.017

25. Chen ZJ, He Y, Rosa P, Germann J, Evans AC. Revealing modular architecture of human brain structural networks by using cortical thickness from MRI. Cereb Cortex (2008) 18:2374-81. doi:10.1093/cercor/bhn003

26. Seeley WW, Crawford RK, Zhou J, Miller BL, Greicius MD. Neurodegenerative diseases target large-scale human brain networks. Neuron (2009) 62:42-52. doi:10.1016/j.neuron.2009.03.024

27. Marin O, Valiente M, Ge XC, Tsai LH. Guiding neuronal cell migrations. Cold Spring Harb Perspect Biol (2010) 2:a001834. doi:10.1101/cshperspect.a001834

28. Mueller SG, Ng P, Neylan T, Mackin S, Wolkowitz O, Mellon S, et al. Evidence for disrupted gray matter structural connectivity in posttraumatic stress disorder. Psychiatry Res (2015) 234:194-201. doi:10.1016/j.pscychresns.2015.09.006

29. Buckley TC, Blanchard EB, Neill WT. Information processing and PTSD: a review of the empirical literature. Clin Psychol Rev (2000) 20:1041-65. doi:10.1016/S0272-7358(99)00030-6

30. Rabinak CA, Angstadt M, Lyons M, Mori S, Milad MR, Liberzon I, et al. Cannabinoid modulation of prefrontal-limbic activation during fear extinction learning and recall in humans. Neurobiol Learn Mem (2014) 113:125-34. doi:10.1016/j.nlm.2013.09.009

31. Hofmann SG. Cognitive factors that maintain social anxiety disorder: a comprehensive model and its treatment implications. Cogn Behav Ther (2007) 36:193-209. doi:10.1080/16506070701421313

32. Herringa R, Phillips M, Almeida J, Insana S, Germain A. Post-traumatic stress symptoms correlate with smaller subgenual cingulate, caudate, and insula volumes in unmedicated combat veterans. Psychiatry Res (2012) 203:139-45. doi:10.1016/j.pscychresns.2012.02.005
33. Helpman L, Marin MF, Papini S, Zhu X, Sullivan GM, Schneier F, et al. Neural changes in extinction recall following prolonged exposure treatment for PTSD: a longitudinal fMRI study. Neuroimage Clin (2016) 12:715-23. doi:10.1016/j.nicl.2016.10.007

34. Sadeh N, Spielberg JM, Miller MW, Milberg WP, Salat DH, Amick MM, et al. Neurobiological indicators of disinhibition in posttraumatic stress disorder. Hum Brain Mapp (2015) 36:3076-86. doi:10.1002/hbm.22829

35. Sadeh N, Spielberg JM, Logue MW, Wolf EJ, Smith AK, Lusk J, et al. SKA2 methylation is associated with decreased prefrontal cortical thickness and greater PTSD severity among trauma-exposed veterans. Mol Psychiatry (2016) 21:357-63. doi:10.1038/mp.2015.134

36. Dedert EA, Green KT, Calhoun PS, Yoash-Gantz R, Taber KH, Mumford MM, et al. Association of trauma exposure with psychiatric morbidity in military veterans who have served since September 11, 2001. J Psychiatr Res (2009) 43:830-6. doi:10.1016/j.jpsychires.2009.01.004

37. Brancu M, Wagner HR, Morey RA, Beckham JC, Calhoun PS, Tupler LA, et al. The post-deployment mental health $(\mathrm{PDMH})$ study and repository: a multisite study of US Afghanistan and Iraq era veterans. Int J Methods Psychiatr Res (2017) 26:e1570-91. doi:10.1002/mpr.1570

38. Kubany ES, Haynes SN, Leisen MB, Owens JA, Kaplan AS, Watson SB, et al. Development and preliminary validation of a brief broad-spectrum measure of trauma exposure: the traumatic life events questionnaire. Psychol Assess (2000) 12:210-24. doi:10.1037/1040-3590.12.2.210

39. Lund M, Foy D, Sipprelle C, Strachan A. The combat exposure scale: a systematic assessment of trauma in the Vietnam war. J Clin Psychol (1984) 40:1323-8. doi:10.1002/1097-4679(198411)40:6<1323::AID-JCLP2270400607>3.0. CO;2-I

40. Beck AT, Steer RA, Brown GK. Manual for the Beck Depression Inventory-II. San Antonio, TX: Psychological Corp (1996).

41. Logue MW, Amstadter AB, Baker DG, Duncan L, Koenen KC, Liberzon I, et al. The psychiatric genomics consortium posttraumatic stress disorder workgroup: posttraumatic stress disorder enters the age of large-scale genomic collaboration. Neuropsychopharmacology (2015) 40:2287-97. doi:10.1038/ npp. 2015.118

42. Fischl B, Van Der Kouwe A, Destrieux C, Halgren E, Segonne F, Salat DH, et al. Automatically parcellating the human cerebral cortex. Cereb Cortex (2004) 14:11-22. doi:10.1093/cercor/bhg087

43. Desikan RS, Segonne F, Fischl B, Quinn BT, Dickerson BC, Blacker D, et al. An automated labeling system for subdividing the human cerebral cortex on MRI scans into gyral based regions of interest. Neuroimage (2006) 31:968-80. doi:10.1016/j.neuroimage.2006.01.021

44. Destrieux C, Fischl B, Dale A, Halgren E. Automatic parcellation of human cortical gyri and sulci using standard anatomical nomenclature. Neuroimage (2010) 53:1-15. doi:10.1016/j.neuroimage.2010.06.010

45. Fjell AM, Walhovd KB, Brown TT, Kuperman JM, Chung Y, Hagler DJ Jr, et al. Multimodal imaging of the self-regulating developing brain. Proc Natl Acad Sci U S A (2012) 109:19620-5. doi:10.1073/pnas.1208243109

46. De Bellis MD, Keshavan MS, Clark DB, Casey BJ, Giedd JN, Boring AM, et al. A.E. Bennett research award. Developmental traumatology. Part II: brain development. Biol Psychiatry (1999) 45:1271-84. doi:10.1016/S00063223(99)00045-1

47. Satterthwaite TD, Vandekar S, Wolf DH, Ruparel K, Roalf DR, Jackson C, et al. Sex differences in the effect of puberty on hippocampal morphology. J Am Acad Child Adolesc Psychiatry (2014) 53:341-50.e341. doi:10.1016/j. jaac.2013.12.002

48. Teicher MH, Anderson CM, Ohashi K, Polcari A. Childhood maltreatment: altered network centrality of cingulate, precuneus, temporal pole and insula. Biol Psychiatry (2014) 76:297-305. doi:10.1016/j.biopsych.2013.09.016

49. Sun D, Peverill MR, Swanson CS, Mclaughlin KA, Morey RA. Structural covariance network centrality in maltreated youth with posttraumatic stress disorder. J Psychiatr Res (2017) 98:70-7. doi:10.1016/j.jpsychires.2017.12.015

50. Rubinov M, Sporns O. Complex network measures of brain connectivity: uses and interpretations. Neuroimage (2010) 52:1059-69. doi:10.1016/j. neuroimage.2009.10.003

51. Winkler AM, Webster MA, Vidaurre D, Nichols TE, Smith SM. Multi level block permutation. Neuroimage (2015) 123:253-68. doi:10.1016/j. neuroimage.2015.05.092

52. Efron B, Tibshirani R. An Introduction to the Bootstrap. New York: Chapman \& Hall (1993). 
53. Zalesky A, Fornito A, Bullmore ET. Network-based statistic: identifying differences in brain networks. Neuroimage (2010) 53:1197-207. doi:10.1016/j. neuroimage.2010.06.041

54. Wang JH, Wang XD, Xia MR, Liao XH, Evans A, He Y. GRETNA: a graph theoretical network analysis toolbox for imaging connectomics. Front Hum Neurosci (2015) 9:386. doi:10.3389/fnhum.2015.00386

55. Northoff G, Bermpohl F. Cortical midline structures and the self. Trends Cogn Sci (2004) 8:102-7. doi:10.1016/j.tics.2004.01.004

56. Lanius RA, Frewen PA, Tursich M, Jetly R, Mckinnon MC. Restoring largescale brain networks in PTSD and related disorders: a proposal for neuroscientifically-informed treatment interventions. Eur J Psychotraumatol (2015) 6:27313. doi:10.3402/ejpt.v6.27313

57. Lanius RA, Bluhm RL, Frewen PA. How understanding the neurobiology of complex post-traumatic stress disorder can inform clinical practice: a social cognitive and affective neuroscience approach. Acta Psychiatr Scand (2011) 124:331-48. doi:10.1111/j.1600-0447.2011.01755.x

58. Litz BT, Stein N, Delaney E, Lebowitz L, Nash WP, Silva C, et al. Moral injury and moral repair in war veterans: a preliminary model and intervention strategy. Clin Psychol Rev (2009) 29:695-706. doi:10.1016/j.cpr.2009. 07.003

59. Currier JM, Holland JM, Jones HW, Sheu S. Involvement in abusive violence among Vietnam veterans: direct and indirect associations with substance use problems and suicidality. Psychol Trauma (2014) 6:73-82. doi:10.1037/ a0032973

60. Frankfurt S, Frazier P. A review of research on moral injury in combat veterans. Mil Psychol (2016) 28:318-30. doi:10.1037/mil0000132

61. Bluhm R, Williamson P, Lanius R, Theberge J, Densmore M, Bartha R, et al. Resting state default-mode network connectivity in early depression using a seed region-of-interest analysis: decreased connectivity with caudate nucleus. Psychiatry Clin Neurosci (2009) 63:754-61. doi:10.1111/j.1440-1819.2009. 02030.x

62. van t'Wout M, Longo SM, Reddy MK, Philip NS, Bowker MT, Greenberg BD. Transcranial direct current stimulation may modulate extinction memory in posttraumatic stress disorder. Brain Behav (2017) 7:e00681. doi:10.1002/ brb3.681

63. Hamani C, Mayberg H, Stone S, Laxton A, Haber S, Lozano AM. The subcallosal cingulate gyrus in the context of major depression. Biol Psychiatry (2011) 69:301-8. doi:10.1016/j.biopsych.2010.09.034

64. Lujan JL, Chaturvedi A, Choi KS, Holtzheimer PE, Gross RE, Mayberg HS, et al. Tractography-activation models applied to subcallosal cingulate deep brain stimulation. Brain Stimulat (2013) 6:737-9. doi:10.1016/j.brs.2013. 03.008

65. Vythilingam M, Nelson EE, Scaramozza M, Waldeck T, Hazlett G, Southwick SM, et al. Reward circuitry in resilience to severe trauma: an fMRI investigation of resilient special forces soldiers. Psychiatry Res (2009) 172:75-7. doi:10.1016/j. pscychresns.2008.06.008

66. Balconi M. Dorsolateral prefrontal cortex, working memory and episodic memory processes: insight through transcranial magnetic stimulation techniques. Neurosci Bull (2013) 29:381-9. doi:10.1007/s12264-013-1309-Z

67. Sun DL, Lee TMC, Chan CCH. Unfolding the spatial and temporal neural processing of lying about face familiarity. Cereb Cortex (2015) 25:927-36. doi:10.1093/cercor/bht284
68. Ochsner KN, Silvers JA, Buhle JT. Functional imaging studies of emotion regulation: a synthetic review and evolving model of the cognitive control of emotion Ann N Y Acad Sci (2012) 1251:E1-24. doi:10.1111/j.1749-6632.2012.06751.x

69. Li W, Qin W, Liu HG, Fan LZ, Wang JJ, Jiang TZ, et al. Subregions of the human superior frontal gyrus and their connections. Neuroimage (2013) 78:46-58. doi:10.1016/j.neuroimage.2013.04.011

70. Hoge CW. Measuring the long-term impact of war-zone military service across generations and changing posttraumatic stress disorder definitions. JAMA Psychiatry (2015) 72:861-2. doi:10.1001/jamapsychiatry.2015.1066

71. Marquez C, Poirier GL, Cordero MI, Larsen MH, Groner A, Marquis J, et al. Peripuberty stress leads to abnormal aggression, altered amygdala and orbitofrontal reactivity and increased prefrontal MAOA gene expression. Transl Psychiatry (2013) 3:e216. doi:10.1038/tp.2012.144

72. Thomaes K, Dorrepaal E, Draijer N, De Ruiter MB, Van Balkom AJ, Smit JH, et al. Reduced anterior cingulate and orbitofrontal volumes in child abuserelated complex PTSD. J Clin Psychiatry (2010) 71:1636-44. doi:10.4088/ JCP.08m04754blu

73. Kroes MCW, Rugg MD, Whalley MG, Brewin CR. Structural brain abnormalities common to posttraumatic stress disorder and depression. J Psychiatry Neurosci (2011) 36:256-65. doi:10.1503/jpn.100077

74. Kolassa IT, Ertl V, Eckart C, Onyut LP, Kolassa S, Elbert T. Spontaneous remission from PTSD depends on the number of traumatic event types experienced. Psychol Trauma (2010) 2:169-74. doi:10.1037/a0019362

75. Morina N, Wicherts JM, Lobbrecht J, Priebe S. Remission from post-traumatic stress disorder in adults: a systematic review and meta-analysis of long term outcome studies. Clin Psychol Rev (2014) 34:249-55. doi:10.1016/j.cpr.2014.03.002

76. Wang T, Wang K, Qu H, Zhou J, Li Q, Deng Z, et al. Disorganized cortical thickness covariance network in major depressive disorder implicated by aberrant hubs in large-scale networks. Sci Rep (2016) 6:27964. doi:10.1038/srep27964

77. Lim L, Radua J, Rubia K. Gray matter abnormalities in childhood maltreatment: a voxel-wise meta-analysis. Am J Psychiatry (2014) 171:854-63. doi:10.1176/appi.ajp.2014.13101427

78. Tijms BM, Series P, Willshaw DJ, Lawrie SM. Similarity-based extraction of individual networks from gray matter MRI scans. Cereb Cortex (2012) 22:1530-41. doi:10.1093/cercor/bhr221

79. Tijms BM, Moller C, Vrenken H, Wink AM, De Haan W, Van Der Flier WM, et al. Single-subject grey matter graphs in Alzheimer's disease. PLoS One (2013) 8:e58921. doi:10.1371/journal.pone.0058921

80. Fornito A, Zalesky A, Bullmore E. Fundamentals of Brain Network Analysis. San Diego: Academic Press (2016).

Conflict of Interest Statement: The authors declare that the research was conducted in the absence of any commercial or financial relationships that could be construed as a potential conflict of interest.

Copyright (C) 2018 Sun, Davis, Haswell, Swanson, Mid-Atlantic MIRECC Workgroup, LaBar, Fairbank and Morey. This is an open-access article distributed under the terms of the Creative Commons Attribution License (CC BY). The use, distribution or reproduction in other forums is permitted, provided the original author(s) and the copyright owner are credited and that the original publication in this journal is cited, in accordance with accepted academic practice. No use, distribution or reproduction is permitted which does not comply with these terms. 